OnLine Journal of Biological Sciences 11 (2): 27-36, 2011

ISSN 1608-4217

(C) 2011 I.M. Pinz et al., This open access article is distributed under a Creative Commons Attribution

(CC-BY) 3.0 license

\title{
Palmitate-Induced Translocation of Caveolin-3 and Endothelial Nitric Oxide Synthase in Cardiomyocytes
}

Catherine J. Knowles, Megan Dionne, Martina Cebova and Ilka M. Pinz

Department of Molecular Medicine, Maine Medical Center Research Institute, 81 Research Drive, ME 04074, Scarborough

\begin{abstract}
Problem statement: Palmitate is a known cardiac lipotoxin that blunts cardiomyocyte contractile function and induces apoptosis, likely via accumulation of the lipotoxic ceramide. Ceramide is a sphingolipid and localizes to caveolae, which are lined in the inner membrane leaflet by caveolin proteins. In this study, we investigated the effects of palmitate on caveolin proteins and on endothelial Nitric Oxide Synthase (eNOS), a signaling mediator that binds to caveolin-3, the musclespecific caveolae scaffolding protein. Approach and Results: Mice fed a high palmitate diet for 12 weeks showed pathologically increased coronary flow in the ex vivo Langendorff heart especially at low extracellular calcium concentrations. In these hearts, eNOS Ser1177 phosphorylation was increased compared to standard or high fat control diet hearts. This suggested that eNOS, a potent vasodilator in the heart, is affected by palmitate. In vitro experiments showed that exposure of HL-1 cardiomyocytes to palmitate causes translocation of eNOS from the plasma membrane to a perinuclear location and causes an $80 \%$ decrease in Thr495 phosphorylation. This corresponded with a $41 \%$ decrease in NO production. To determine the mechanism of the loss of plasma membrane bound eNOS, we investigated the effect of palmitate on caveolin-3 and found decreased caveolin-3 protein levels by $70 \%$ compared to control cells. The remaining $30 \%$ of caveolin-3 was localized to a perinuclear location. In contrast to previous studies, palmitate did not cause apoptosis in cardiomyocytes. Conclusion: Overall, we show for the first time that a high palmitate diet leads to loss of caveolin-3 in cardiomyocytes and to coronary dysfunction of the mouse heart, via uncoupling of eNOS.
\end{abstract}

Key words: Endothelial Nitric Oxide Synthase (eNOS), palmitate, sphingolipids, coronary flow, caveolin scaffolding domain, lipotoxic ceramide, fatty acids, cardiomyocytes

\section{INTRODUCTION}

Morbidities associated with obesity such as atherosclerosis, hypertension and diabetes are positively correlated to plasma fatty acid content. Cardiac lipotoxicity, caused by the accumulation of lipids, is observed in rodent models of obesity, hyperlipidemia and diabetes (Zhou et al., 2000; Chiu et al., 2001; Young et al., 2002; Yagyu et al., 2003; Chiu et al., 2005). To define the role of fatty acids, several studies focused on saturated versus unsaturated fatty acids, of isolated cardiomyocytes (Fauconnie et al., 2007; Okere et al., 2006). However, the direct effect of a diet rich in palmitate (the most dominant fat in the western fast food diet) on cardiac caveolin proteins and the consequences for caveolin binding proteins has not yet been established.

Exposing isolated cardiomyocytes to high concentrations of the saturated fatty acid palmitate cardiac substrate utilization and contractile performance

induces contractile dysfunction and apoptosis (Kong and Rabkin. 2003; Tsang et al., 2004; Miller et al., 2005; Fauconnier et al., 2007). Most previously published studies suggest that accumulation of ceramide is the mediator of palmitate's deleterious effects. Ceramide is a sphingolipid, which is enriched in micro-domains of the plasma membrane called caveolae (Anderson, 1998). Caveolae are flask-like membrane invaginations, $50-100 \mu \mathrm{m}$ in size and function in endocytosis, lipid trafficking and signal transduction (Anderson, 1998; Okamoto et al., 1998). There are three caveolin isoforms, caveolin-1, -2 and 3. Caveolin-1 and -2 are ubiquitously expressed, whereas caveolin-3 is specific for striated muscle (Williams and Lisanti, 2004). Caveolin-1 and -3 have a conserved Caveolin Scaffolding Domain (CSD), which is bound by many membrane proteins, for example Gproteins, the EDG receptors, tyrosine kinase receptors, Erk and eNOS (Gratton et al., 2004). Thus, caveolae play a central role in cellular communication and signal Corresponding Author: Ilka M. Pinz, Department of Molecular Medicine, Maine Medical Center Research Institute, 81 Research Drive, ME 04074, Scarborough Tel: 2073968280 Fax: 2073968179 
transduction, providing tight and efficient regulation of intracellular cascades.

In cardiomyocytes, eNOS localizes to caveolae (Garcia-Cardena et al., 1996; Feron and Balligand, 2006) binding to caveolin-3. The co-localization of caveolin-3 and eNOS may facilitate both eNOS activation by cell surface receptors and nitric oxide (NO) release at the cell surface for intercellular signaling (Feron and Balligand, 2006). The first identified mechanism for membrane association of eNOS was lipid modification: eNOS is the only NOS that undergoes both myristoylation and palmitoylation on glycine 2 and cysteines 15 and 26. This double acylation is necessary for the targeting of eNOS to plasmalemmal caveolae (Feron and Balligand, 2006). In cardiac ischemia preconditioning, release of eNOS from caveolin is a crucial step for normal function of eNOS and the protective release of NO (Der et al., 2006). It is unknown whether in vivo exposure to palmitate has an effect on eNOS binding to caveolae. Therefore, we tested the hypothesis that exposure to high palmitate changes the association of eNOS with caveolin-3 in cardiomyocytes, independent of induction of apoptosis. We present evidence for the first time that a diet high in palmitate leads to increased cardiac eNOS activation and pathological changes in cardiac coronary blood flow in vivo. This is further supported by in vitro studies, showing palmitate-induced loss of membrane localized caveolin-3 and the translocation of eNOS away from the plasma membrane, which in turn is associated with a change in phosphorylation status and activity of the enzyme.

\section{MATERIALS AND METHODS}

Animals: C57BL/6 mice were purchased from Taconic Farms. After breeding at MMCRI weaned mice (3 weeks of age) were maintained on standard lab chow (6\% crude fat) or a customized high palmitate diet (palmitate, Harlan Teklad, TD05235, 21\% total fat of which $47 \%$ is palmitate) for 12 weeks. As a high fat control diet, a customized diet containing no palmitate and only medium chain triglycerides was used (MCT diet, Harlan Teklad, TD05237, 21\% of total fat). Experimental protocols and procedures involving animals were approved by the Institutional Animal and Use Committee of Maine Medical Center and followed the recommendations of current National Institutes of Health and American Physiological Society guidelines for the use and care of laboratory animals.

Isolated, Isovolumic Cardiac Langendorff perfusion: Mice were anesthetized with Avertin $(0.3$ $\mathrm{mg} \mathrm{g}^{-1}$ ) and euthanized by cervical dislocation. Hearts were quickly excised and perfused in the Langendorff mode following previously published procedures (Pinz et al., 2008). After establishing baseline cardiac contractile performance, hearts were challenged by increasing extracellular calcium concentration from 1.5 - $4 \mathrm{mM}$, in $0.5 \mathrm{mM}$ steps, by adding a $50 \mathrm{mM}$ calcium solution to the perfusate at 1,2 and 3\% of coronary flow with a non-pulsatile pump. Coronary flow was measured with an in-line flow sensor (1PXN, Transonic Systems, Inc.). The calcium challenge will increase coronary flow in a linear fashion in a normal heart.

Cell culture: The HL-1 cell line is a cardiomyocyte line derived from the AT-1 mouse atrial cardiomyocyte lineage and established and previously described by Claycomb et al. (1998). Prior to culture of cells, dishes were coated with $0.5 \%$ bovine fibronectin (Sigma) in $0.02 \%$ gelatin overnight at $37^{\circ} \mathrm{C}$. HL-1 cells were cultured in Claycomb's specific media (SAFC Biosciences) supplemented with $10 \%$ fetal bovine serum (SAFC Biosciences), $0.1 \mathrm{mM}$ norepinephrine, 2 $\mathrm{mM}$ L-glutamine, 100 units $\mathrm{mL}^{-1}$ penicillin and $100 \mu \mathrm{g}$ $\mathrm{mL}^{-1}$ streptomycin and grown at $37^{\circ} \mathrm{C}$ in an atmosphere of $5 \% \mathrm{CO}_{2}$ and $95 \%$ air. Cultures were grown to $75 \%$ confluency before treatment and $100 \%$ confluency before being passaged or harvested for analysis.

Cell treatments: After culture for $24 \mathrm{~h}(30-50 \%$ confluency) in supplemented Claycomb's medium, cells were washed with serum-free Claycomb's medium and cultured in serum-free Claycomb's medium for 12$24 \mathrm{~h}$ prior to treatment with experimental conditions. To simulate the components of Claycomb's medium, while maintaining control of BSA-bound palmitate, the following components were added to Dulbecco's Modified Essential Media (DMEM) creating a basal serum-free medium: $2 \mathrm{mg} \mathrm{mL} \mathrm{mL}^{-1} \mathrm{BSA}, 2 \mathrm{mM} \mathrm{L}-$ carnitine, $5 \mathrm{mM}$ creatine, $5 \mathrm{mM}$ taurine, $1 \mathrm{mM} \mathrm{L}$ glutamine, 100 units $\mathrm{mL}^{-1}$ penicillin, $100 \mu \mathrm{g} \mathrm{mL}^{-1}$ streptomycin, $0.1 \mathrm{mM}$ norepinephrine (added fresh day of use). Control medium was comprised of this medium and contained $5 \%$ fatty acid free BSA only, whereas experimental medium contained $0.4 \mathrm{mM}$ palmitate bound to $5 \%$ fatty acid free BSA. Culture medium containing 0.1 or $0.2 \mathrm{mM}$ palmitate was made by dilution of $0.4 \mathrm{mM}$ palmitate medium with control medium. Cells were exposed to control, $0.1,0.2$ or 0.4 $\mathrm{mM}$ palmitate medium for $18 \mathrm{~h}$.

Cell transfections: After culturing to $80 \%$ confluency in $100 \mathrm{~mm}$ culture dishes, HL-1 cells were placed in 
antibiotic free supplemented Claycomb's medium for 4 $\mathrm{h}$ prior to transfection. Cells were transfected with Lipofectamine 2000 (Invitrogen) following the recommendations of the manufacturer using $4 \mu \mathrm{g}$ of plasmid DNA encoding bovine eNOS (a generous gift from T. Michel at Brigham and Womens Hospital, Boston, MA). Transiently transfected cells were identified by co-transfection of GFP and to provide an estimate of transfection efficiency.

Sucrose gradient centrifugation: Isolation of caveolae-containing insoluble lipid domains of HL-1 cardiomyocytes was performed using sucrose gradient centrifugation as previously described by Song et al., (1996). After centrifugation, ten $1.2 \mathrm{~mL}$ fractions were collected from the top of the gradient. Each fraction was centrifuged at $10,000 \times \mathrm{g}$ for $15 \mathrm{~min}$ at $4^{\circ} \mathrm{C}$ and the pellet was resuspended in $50 \mathrm{mM}$ Tris, $\mathrm{pH} 8.8$ with 2 $\mu \mathrm{g} \mathrm{mL}^{-1}$ aprotinin. Equal amounts of the resulting samples were dissolved in SDS-Polyacrylamide Gel Electrophoresis (PAGE) loading buffer for determination of caveolin proteins and eNOS.

Confocal microscopy: HL-1 cells were grown on fibronectin-gelatin coated glass coverslips and treated in the same manner as described above. After treatment, cells were washed twice in ice cold PBS and fixed with 4\% paraformaldehyde in PBS for $10 \mathrm{~min}$ at RT. Coverslips were washed with ice cold PBS and stored in PBS with $0.01 \%$ sodium azide. Fixed cells were blocked and permeabilized with 5\% BSA, $0.1 \%$ Triton$\mathrm{X}-100,0.1 \%$ Tween 20 and $0.1 \%$ sodium azide in PBS. Primary caveolin-3 antibody (BD Transduction \#610420) was diluted in PBS with 1\% BSA and 0.3\% Triton-X-100. Primary antibodies were incubated at $4^{\circ} \mathrm{C}$ overnight followed by incubation with fluorescently labeled secondary antibodies. Alexa Fluor 488 labeled goat anti-mouse IgG (Invitrogen) and Alexa Fluor 546 goat anti-mouse IgG (Invitrogen) were incubated for 1 hour at RT. Cells were counter stained with the DNA stain TOPRO for $30 \mathrm{~min}$ at RT directly after secondary antibody incubation. Cells were mounted with ProLong Gold anti-fade reagent (Invitrogen), allowed to cure overnight at RT, sealed and examined with a Leica TCS SP II True Confocal Laser Scanning Microscope and analyzed using the Leica confocal software.

Western blot analysis: After experimental treatment, cells were lysed in the presence of a phosphatase and protease inhibitor solution of the following composition: $50 \mathrm{mM} \mathrm{NaF}, 10 \mathrm{mM} \beta$-glycerolphosphate, $1 \mathrm{mM}$ sodium orthovanadate, $10 \mathrm{mM}$ sodium pyrophosphate and protease inhibitor cocktail V (Calbiochem). Protein concentration was determined according to Lowry et al. (1951). Equal amounts of sample proteins were subjected to SDS-PAGE and transferred to nitrocellulose membranes. Primary antibodies used were: anti-eNOS (BD Transduction Laboratories), anti-peNOSSer1177 (BD Transduction Laboratories), anti-peNOS Thr495 (Cell Signaling), anti-caveolin-3 (BD Transduction Laboratories), anticaspase-3 (Cell Signaling) and anti-GAPDH (Santa Cruz). Chemiluminescent HRP antibody detection reagent was used (Denville Scientific) and blots were scanned and analyzed using NIH ImageJ software.

eNOS activity assay: The Nitric Oxide (NO) producing activity of eNOS was assessed in HL-1 cardiomyocytes using the kit available from Oxford Biomedical Research Inc. following the manufacturer's recommendations.

Data analysis and statistics: Data are presented as means \pm SE. One-way ANOVA or regression analysis was performed using GraphPad Prism (GraphPad Prism 4.0, GraphPad software, San Diego, CA) were appropriate.

\section{RESULTS}

Palmitate feeding of mice increases eNOS $^{\text {Ser1177 }}$ phosphorylation and increases coronary flow in adult mice: Feeding C57BL6 mice a high palmitate diet for 12 weeks leads to persistent increased coronary flow over a wide range of extracellular calcium concentrations (Fig. 1A). Especially at low extracellular calcium the pathological increase to $16 \mathrm{~mL} \mathrm{~min}^{-1} * \mathrm{~g}_{\mathrm{LV}}$ in coronary flow is apparent. This suggests that a strong vasodilator, such as the Nitric Oxide Synthases (NOS), is activated by the palmitate diet. NOS produce NO, a potent vasodilator and in the heart endothelial NOS (eNOS) is expressed in the cardiac endothelium and in cardiomyocytes. To test its involvement in the palmitate mediated effect in vivo; we measured phosphorylation of eNOS in standard, MCT and palmitate diet fed mice. Consistent with the hypothesis, the phosphorylation of

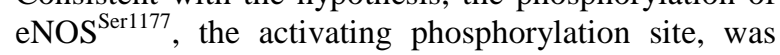
increased in mice fed a high palmitate diet (Fig. 1B and C). Phosphorylation at Thr495, the inhibitory site, was not detectable in the in vivo samples. To further evaluate the mechanism of how palmitate affects eNOS activity, we investigated its effect on the expression and localization of caveolin-3, the striated muscle specific isoform of caveolin and to which eNOS binds at the plasma membrane. 


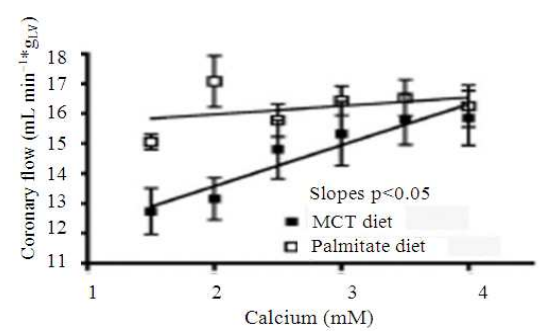

(a)

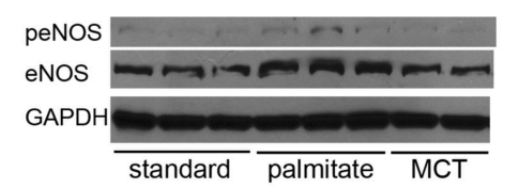

(b)
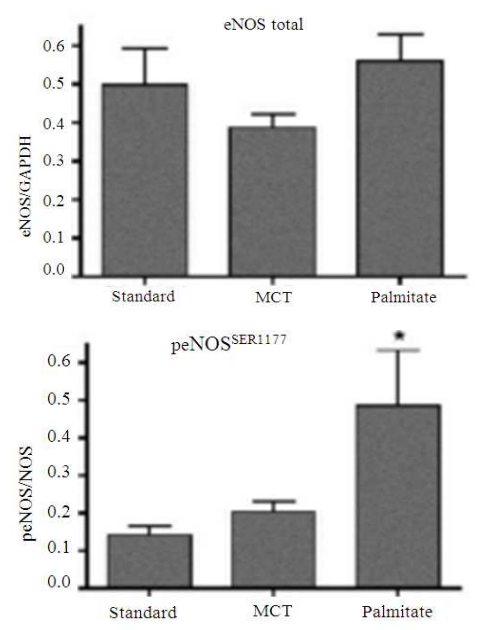

(c)

Fig. 1: Palmitate increases coronary flow and

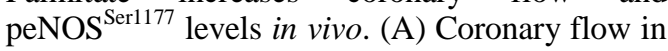
isolated Langendorff perfused hearts after 12 weeks on control or palmitate diet (standard diet values are equal to control diet values). Hearts from palmitate fed mice had increased coronary flow, which was particularly distinct at low extracellular calcium concentrations. Means \pm SE, $n=4-8$, linear regression analysis performed in GraphPad Prism 4.0. (B) eNOS and peNOS $^{\text {Ser1177 }}$ protein in left ventricular tissue was analyzed by immunoblot. Ventricular lysates were obtained from heart tissue after 12 weeks of standard, MCT or palmitate diet. GAPDH was used as loading control. (C) Quantification of immunoblots showing no change of total eNOS expression (eNOS/GAPDH), but a 5fold increase of Ser1177 phosphorylation in heart tissue of palmitate diet fed mice compared to standard or MCT diet fed mice. Means \pm SE, $N=5-6, * p<$ 0.05 one-way ANOVA
Palmitate-induced loss of membrane bound eNOS in HL-1 cardiomyocytes: HL-1 cells currently provide the only opportunity to utilize an immortalized cardiac cell line for in vitro experiments. When eNOS transfected HL-1 cardiomyocytes (these cells do not express endogenous eNOS) were treated with varying concentrations of palmitate, we observed changes in the subcellular eNOS localization. Compared to control cells, in cultures treated with $0.4 \mathrm{mM}$ palmitate eNOS moves from the plasma membrane to the peri-nuclear area (Fig. 2A). Interestingly, when HL-1s are exposed to palmitate in the presence of myriocin - a natural mycotoxin that inhibits the first step of the de-novo synthesis of ceramide from palmitate (He et al., 2004; Johnson et al., 2004)-most cells retain proper localization of eNOS to the plasma membrane. These results suggest that the de novo synthesis of sphingolipids from palmitate is a regulator of subcellular localization of eNOS.

In order to determine what subcellular compartments are affected by the changes in eNOS localization, we subjected lysates of HL-1 cultures to sucrose gradient centrifugation. These results also confirm the loss of eNOS from the caveolar fraction and its movement to different membrane systems of the cell (Fig. 2B). In control treated cells, eNOS localizes to the higher buoyant fractions (fraction 4)-indicating its location in the plasma membrane. Upon treatment with palmitate, eNOS has a trend toward moving to lower buoyant fractions (Fractions 7 -10)-indicating its movement to more dense membranes such as nuclear, ER or the Golgi apparatus. In the higher-buyoant fractions, palmitate induces a 6-fold loss of eNOS and in the lower buoyant fractions, there is a 3 -fold increase in eNOS levels. These results exhibit high variability, likely an inherent artifact of transfection as this leads to overexpression of the protein.

Concomitant with the translocation of eNOS, the NO producing activity and the phosphorylation status changes (Fig. 2C and D). eNOS can be phosphorylated at two regulatory sites: The inhibitory Thr495 and the activating Ser1177. Palmitate exposure decreases the phosphorylation of eNOS at Thr495 (Fig. 2C), suggesting that translocation makes this site allosterically available for dephosphorylation by phosphatases. To determine how loss of Thr495 phosphorylation affects NO production in HL-1 cells, we determined eNOS activity using a colorimetric kit that measures nitrate production from NO (Fig. 2D). NO release was equal between HL-1 cells exposed to control, $0.1,0.2 \mathrm{mM}$ palmitate. In $0.4 \mathrm{mM}$ palmitate exposed cells NO synthesis was decreased by $41 \%$. This suggests that palmitate-induced dephosphorylation at Thr495 leads to uncoupling of eNOS with lower output of NO in HL-1 cardiomyocytes. 

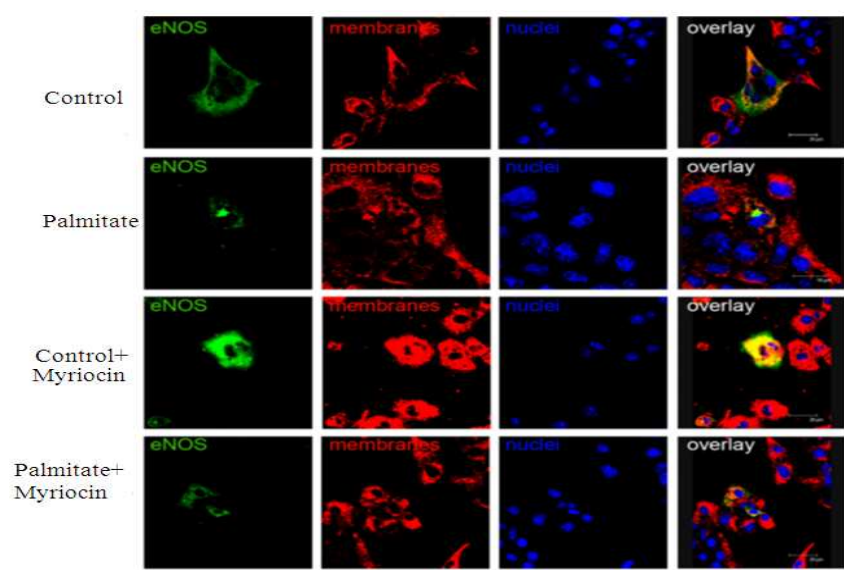

(a)

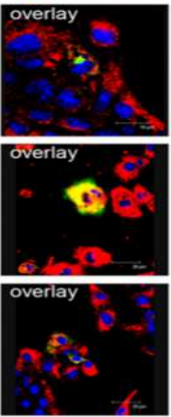

$-$

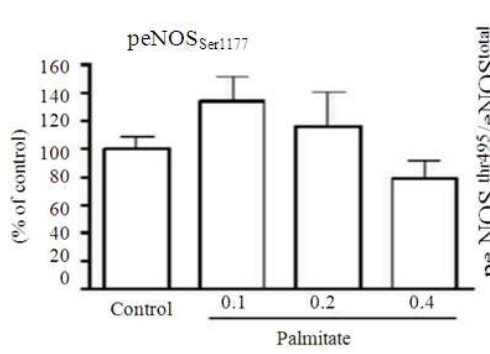

(c)

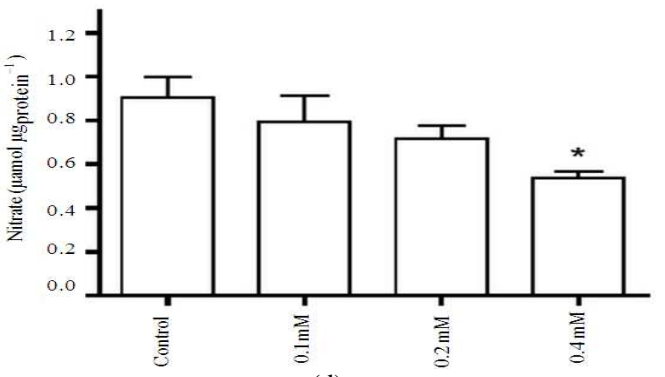

(d)

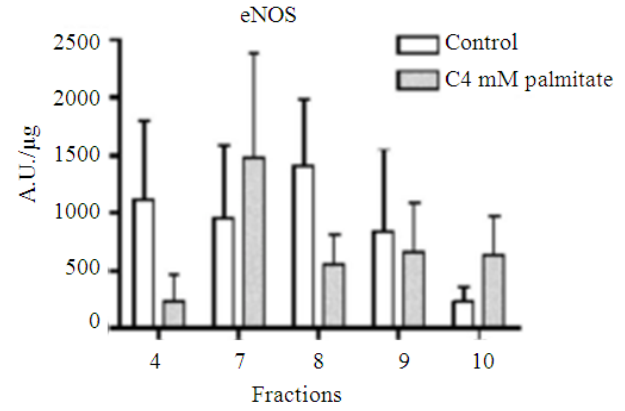

(b)
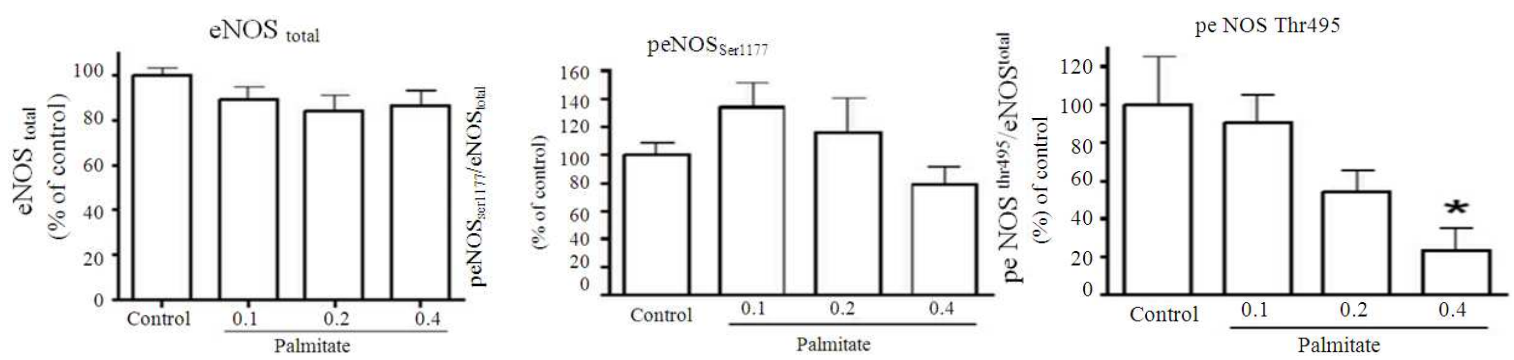

Fig. 2: Palmitate induces translocation of cellular eNOS and its differential phosphorylation in HL-1 cardiomyocytes. (A) Representative confocal images of eNOS localization in HL-1 cardiomyocytes under control and palmitate $(0.4 \mathrm{mM})$ conditions. In addition, cells were treated with an inhibitor of the de novo ceramide synthesis pathway, myriocin $(5 \mu \mathrm{M})$. Cells treated with control conditions show localization around the cell periphery, while treatment with palmitate causes movement to the cell's interior. Green $=\mathrm{eNOS}$, Red $=$ lipid, Blue $=$ DNA, Yellow = areas of eNOS/lipid colocalization. (B) Quantification of eNOS in sucrose gradients from cell lysates of control or $0.4 \mathrm{mM}$ palmitate treated cells. In palmitate treated cells eNOS localizes less to the caveolar fraction 4, but more to the low buoyant nuclear membrane fractions (fraction 10), similar to the caveolin-3 distribution. (C) eNOS is phosphorylated differentially at two phosphorylation sites. Phosphorylation of Ser1177, the activating site, is slightly increased with $0.1 \mathrm{mM}$ and $0.2 \mathrm{mM}$ palmitate, but falls to normal levels upon treatment of $0.4 \mathrm{mM}$ palmitate. In contrast, phosphorylation at the inhibitory site, Thr 495, is significantly decreased upon treatment with higher palmitate concentrations. Means $\pm \mathrm{SE} n=3, \mathrm{p}<0.05$ onefactor ANOVA. (D) Activity of eNOS in cells exposed to control or $0.1 \mathrm{mM}, 0.2 \mathrm{M}$ or $0.4 \mathrm{mM}$ palmitate medium. The release of NO decreases with increasing palmitate concentration, but reaches significance only at $0.4 \mathrm{mM}$ palmitate. Means $\pm \mathrm{SE}, \mathrm{n}=8, * \mathrm{p}<0.02$ one-factor ANOVA 
OnLine J. Biol. Sci., 11 (2): 27-36, 2011

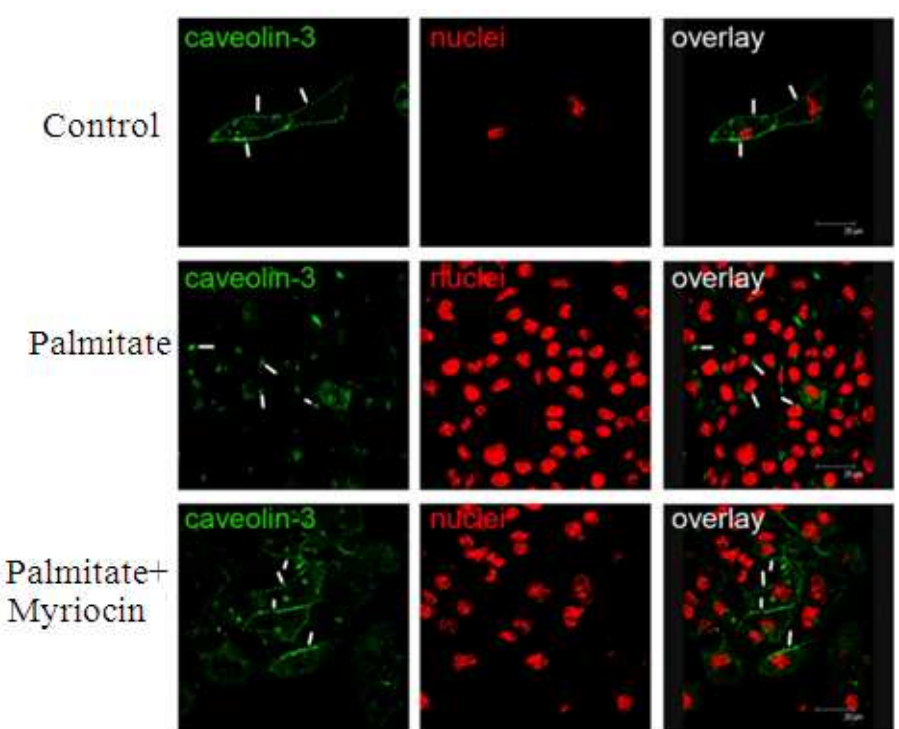

(a)

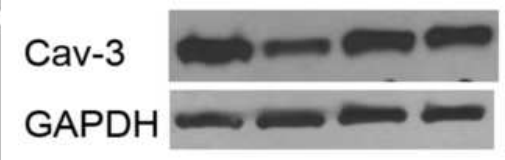

MG-132 $\frac{+\quad-}{\text { palmitate }} \frac{+\quad-}{\text { control }}$

(b)

Fig. 3: Palmitate-induces translocalization of caveolin-3 from the plasma membrane to the peri-nuclear area. (A) Confocal images of HL-1 cells exposed to control medium or $0.4 \mathrm{mM}$ palmitate medium for $18 \mathrm{~h}$. Under control conditions, caveolin-3 is associated with the plasma membrane. In contrast, palmitate induces translocalization of caveolin-3 away from the plasma membrane to the peri-nuclear area (arrows). This translocalization can be prevented by inhibiting the first step of the de-novo synthesis of ceramide with myriocin. Green = Caveolin-3, Red = DNA. Scale different in first panel to show plasma membrane localization of caveolin-3 in two adjacent cells. (B) Proteins isolated from the sucrose gradient were analyzed by immunoblot to detect caveolin-3 in sub-cellular fractions. Palmitate induces movement of caveolin-3 away from the plasma membrane (the high buoyant caveolae fractions (4) to less-buoyant membrane fractions (fractions 810) in a concentration dependent manner. (C) Quantitative analysis of the western shown in panel B. Experiments were repeated three times with similar results. (D) Inhibition of protein degradation by MG132 (10 $\mu \mathrm{M})$ prevents the palmitate-induced decrease of caveolin-3 protein

Palmitate-induces translocation of caveolin-3 from the plasma membrane to the peri-nuclear area and enhances degradation - Caveolin-1 is a known important regulator of eNOS function (Feron et al., 1998; Feron and Balligand, 2006). In order to determine the effect of palmitate on caveolin-3 protein we exposed HL-1 cardiomyocytes to $0.1,0.2$, or 0.4 $\mathrm{mM}$ palmitate. Figure $3 \mathrm{~A}$ shows that palmitate exposure changes caveolin-3 localization in HL-1 cardiomyocytes. As expected, in control mediumtreated cells the localization of caveolin-3 (green) coincides with the plasma membrane of the cardiomyocyte. In contrast, treatment with $0.4 \mathrm{mM}$ palmitate leads to a loss of membrane bound caveolin-3 and localization to a peri-nuclear location. We observed a clear concentration dependence of this process as 0.1 , $0.2 \mathrm{mM}$ palmitate exposed cells show intermediate localization to the plasma membrane and the perinuclear area (data not shown). Interestingly, upon treatment with myriocin, palmitate exposed HL-1 cells maintained most of their caveolin-3 at the plasma membrane. This links the palmitate-induced translocation of caveolin-3 to the palmitate-induced accumulation of sphingolipidsInhibition of protein ubiquitination and proteasomal degradation with the inhibitor MG-132 shows that caveolin-3 is degraded rapidly after it loses its plasma membrane localization (Fig. 3D). Thus, we cannot exclude that at least some of the caveolin-3 protein observed in the nuclear local in the confocal images is protein in the process of translation in the ER.

Palmitate does not induce apoptosis in HL-1 cardiomyocytes - It is well known that palmitate exposure can induce apoptosis (Kong and Rabkin. 2003; Kong and Rabkin. 2004; Miller et al., 2005). In contrast to previous studies, palmitate did not induce apoptosis in our experimental conditions. The cleavage and activation of caspase 3 (MW $17 \mathrm{kDa}$ ) was not detected by Western blot analysis (Fig. 4). 
OnLine J. Biol. Sci., 11 (2): 27-36, 2011

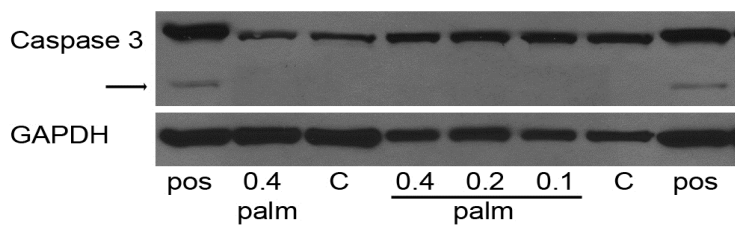

Fig. 4: Palmitate does not induce apoptosis in HL-1 cardiomyocytes. Western blot showing the expression of uncleaved caspase 3 in control (C) and $0.4 \mathrm{mM}, 0.2 \mathrm{mM}$ and $0.1 \mathrm{mM}$ palmitate exposed HL-1 cells. Arrow points to positive control (pos). GAPDH is shown as loading control. Experiment was repeated 4 times with the same result

This excludes that the translocation of caveolin-3 is caused by the induction of apoptosis and the degradation of cellular membrane systems.

\section{DISCUSSION}

It is well known that palmitate causes lipotoxic changes in the heart (Kong and Rabkin. 2003; Tsang et al., 2004; Miller et al., 2005; Fauconnier et al., 2007). The accumulation of lipotoxic ceramide from de novo synthesis is considered one of the main mechanisms for induction of cardiomyocyte apoptosis (Okere et al., 2006). Here we present data that suggests a mechanism for lipotoxic changes that are independent of apoptosis. This mechanism includes the loss of caveolin- 3 from the plasma membrane of cardiomyocytes and the concomitant loss of caveolin-3 binding proteins, in particular eNOS, from the plasma membrane.

Diet induced changes in coronary flow: Our in vivo mouse model is, to our knowledge, the first to show early pathological effects of a high saturated fat diet on coronary flow. Feeding a high palmitate diet for 12 weeks is sufficient to maximize coronary flow at low cardiac workloads. We suggest that the diet-induced increase in coronary flow is possibly due to the up regulation of a potent cardiac vasodilator. There are three potent vasodilators in the heart, adenosine, NO and acidic $\mathrm{pH}$. Adenosine is produced by the breakdown of ATP in the presence of increased ATP demand but insufficient ATP supply (Ingwall, 2002). A subsequent adenylate kinase reaction utilizes 2 ADP molecules to generate one ATP and one AMP molecule. The AMP is quickly degraded to adenosine, which exits the cell via its concentration gradient. It binds to $\mathrm{G}$ protein coupled adenosine receptors in the endothelium. This results in vasodilatation and increased coronary flow (Mubagwa and Flameng,
2001). While we cannot exclude that some of the palmitate-induced increase in coronary flow is due to adenosine formation, we were not able to detect significant increases in adenosine concentrations in the heart or loss of ATP (both measured by HPLC, data not shown). Another strong vasodilator in the heart is NO. It is produced by eNOS and iNOS in the endothelium and in cardiomyocytes. We have shown a 5-fold increase in eNOS expression in the hearts of mice fed a high palmitate diet, with no change in iNOS (data not shown). Thus, it is likely that in vivo the palmitate dietinduced increased coronary flow is caused by increased NO production, despite the decreased NO production we have observed in vitro. The heart consists of about $20 \%$ cardiomyocytes, but they account for more than half of the total cellular mass of the heart. The remaining cells are fibroblasts and endothelial cells. The observed difference between in vivo and in vitro effects of palmitate may be due to different effects of palmitate on endothelium, fibroblasts, or cardiomyocytes. It is conceivable that concentration effects also play an important role. We know from mass spectrometric analysis that the free palmitate concentration in the blood plasma of our high palmitate diet fed mice is $0.1 \mathrm{mM}$ (data not shown). This accounts only for the unbound palmitate, thus using 0.4 $\mathrm{mM}$ palmitate in our in vitro experiments approximates the total concentration present in vivo. We cannot exclude that cardiomyocytes in vivo are exposed to lower amounts because the endothelium regulates the amount of palmitate uptake and thus the amount, which is available to the cardiomyocyte. Thus, the endothelium in vivo might show deleterious effects, such as ROS production, earlier than cardiomyocytes. This may also explain the increase in eNOS expression we have observed in vivo, which could be a compensatory response to declining NO levels. In addition, increased NO release in vivo can inhibit oxidative mitochondrial respiration (Kanai et al., 2001), thus energy metabolism has to rely on anaerobic metabolism. This could lead to a drop in intracellular $\mathrm{pH}$, another strong factor contributing to vasodilation in the heart.

To further define the mechanisms of palmitateinduced changes in eNOS, its phosphorylation state and activity, we investigated the link between palmitate exposure and eNOS activity and phosphorylation status in an in vitro system using HL-1 cardiomyocytes.

Palmitate-induced translocalization of eNOS and differential phosphorylation - Intracellular eNOS localizes to caveolae and binds the CSD domain of caveolin-1 in endothelial cells (Feron et al., 1998). This 
study suggests that the binding of caveolin inhibits eNOS activity by preventing calmodulin binding to the calmodulin-binding domain of eNOS (Feron et al., 1998). Upon release of caveolin, eNOS moves away from the plasma membrane, calmodulin binds to eNOS activating production of NO. In contrast, other reports have suggested that the binding of caveolin proteins stabilizes the membrane localization of eNOS and enables eNOS to produce NO where it is needed (Liu et al., 1996). In this study, we have found that palmitate exposure of cardiomyocytes translocates both caveolin-3 and eNOS away from the plasma membrane and both proteins localize to a peri-nuclear location. Translocation seems likely, but we cannot exclude that palmitate treatment affects membrane composition or function. If for example the lipid composition of the ER membrane was changed, protein export may be impaired leading to accumulation of proteins in the ER. The decrease in plasma membrane association of caveolin-3 and eNOS may then represent normal plasma membrane protein turnover that is not replaced due to the defective ER-Golgi protein export in the presence of excess palmitate.

Our results do not indicate whether caveolin-3 and eNOS remain bound to each other in the peri-nuclear location. However, because the phosphorylation status of eNOS changes upon translocation to the peri-nuclear area, we suggest that eNOS is not associated with caveolin-3. Phosphorylation at Ser1177 does not change, however phosphorylation at Thr495 decreases and cellular release of NO from HL-1 cells decreases by $41 \%$. This suggests that binding of caveolin- 3 blocks access to Thr495 in the calmodulin-binding-domain of eNOS and protects it from phosphatases. Phosphatase access to this site also suggests that calmodulin, an important enhancer of eNOS activity, is not bound to the protein. Some reports have shown a link between high sphingolipid levels and increased intracellular calcium concentrations, which could increase calmodulin expression and possibly enhance binding to eNOS. We did not find increased calmodulin expression in hearts of mice fed a high palmitate diet (data not shown) and the decrease in released NO in vitro also suggests that calmodulin is not bound to eNOS. Thus, we conclude that in vitro exposure of cardiomyocytes to palmitate leads to release of eNOS and caveolin-3, prevention of calmodulin binding to eNOS, followed by the uncoupling of eNOS with decreased synthesis of NO. Whether this leads to an increase in ROS production remains to be determined.

Palmitate-induced loss of plasma membrane caveolin-3 as a novel mechanism for regulation of
eNOS activity: The plasma membrane contains small membrane micro-domains called caveolae. The loss of caveolin-3, the structural protein of caveolae, in mice induces cardiomyopathy (Woodman et al., 2002) and muscular dystrophy (Minetti et al., 1998). Here we report for the first time, that palmitate exposure is sufficient to induce the loss of caveolin-3 from the plasma membrane in vitro. With palmitate exposure, caveolin-3 translocates to a peri-nuclear local. It seems likely that it localizes to the Golgi membrane system, because it is closely associated with the nucleus. As a possible mechanism we suggest that palmitate, as reported in the literature, increases cellular sphingomyelin and ceramide levels and that this sphingolipid accumulation changes the structure of membrane micro-domains. Our data strongly suggest that palmitate exposure leads to the loss of caveolin-3, via enhanced protein degradation via the ubiquitinproteasome pathway and that this affects downstream signaling cascades that localize and bind to caveolin proteins, as we have shown for eNOS in this study. This is a novel finding for the regulation of eNOS activity. Figure 3 indicates that caveolin-3 does not localize to endosomes or lysosomes, however inhibition of the ubiquitin-proteasome pathway with MG-132 (Fig. 3D) suggests that increased protein breakdown accounts for the loss of caveolin-3. We cannot exclude that the caveolin-3 found in the peri-nuclear area is in the process of translation and processing for translation to the plasma membrane. The accumulation of sphingolipids as a result of high palmitate may change the lipid composition of the ER-Golgi system in such a way as to perturb normal protein transport processes.

In contrast to other studies, we have not been able to demonstrate increased apoptosis in cells exposed to palmitate (Kong and Rabkin. 2003; 2004; Miller et al., 2005). Even with the highest concentration used (0.4 $\mathrm{mM})$, the cells did not show activation of caspase 3 (Fig. 4). This strongly suggests that the change in translocation of caveolin-3 is not due to apoptotic changes in the cell but rather a direct effect of palmitate exposure.

Difference between in vivo and in vitro effects of palmitate: We have found increased coronary flow in mice fed a palmitate diet. Interestingly, our in vitro data do not show an increased NO release from cardiomyocytes in a high palmitate environment. The observed difference between in vivo and in vitro effects of palmitate may be due to different effects of palmitate on endothelium and cardiomyocytes. The heart consists to about $20 \%$ of cardiomyocytes, but they account for more than half of the total cellular mass. The remaining 
cells are fibroblasts and endothelial cells. It is conceivable that concentration effects also play an important role. We know from mass spectrometric analysis that the free palmitate concentration in the blood plasma of high palmitate diet fed mice is $0.1 \mathrm{mM}$ (data not shown). Thus using $0.4 \mathrm{mM}$ palmitate in our in vitro experiments approximates the total concentration present in vivo. We cannot exclude that cardiomyocytes in vivo are exposed to lower amounts because the endothelium buffers the amount and takes up most of the available palmitate. Thus, the endothelium in vivo might show deleterious effects, such as ROS production, earlier than cardiomyocytes and may contribute to the phenotype seen in ex vivo hearts.

\section{CONCLUSION}

In summary, the finding of palmitate-induced loss of caveolin-3 in cardiomyocytes has significant implications for populations that eat a western style high fat diet. The increasing incidence of obesity in the adult and adolescent population has also increased the co-morbidity of cardiovascular diseases. Our data suggest that a high palmitate dietary intake will over time lead to changes in the micro-domain structure of the cardiomyocytes plasma membrane and the loss of the structural component of caveolae, the caveolin protein(s). This may negatively impact associated signaling pathways, as we have shown for eNOS and NO production. We suggest that these changes in membrane composition in the long term contribute to the onset of obesity-associated co-morbidities.

\section{ACKNOWLEDGEMENT}

Study was supported by grants from the National Center for Research Resources (NCRR), a component of the National Institutes of Health (5 P20 RR15555-10, subproject 6 to IP). Its contents are solely the responsibility of the authors and do not necessarily represent the official views of NCRR or NIH; and by the American Heart Association (0930260N to IP).

\section{REFERENCES}

Anderson, R.G., 1998. The caveolae membrane system. Annu. Rev. Biochem, 67: 199-225. DOI: 10.1146/annurev.biochem.67.1.199

Chiu, H.C., A. Kovacs, D.A. Ford, F.F. Hsu and R. Garcia et al., 2001. A novel mouse model of lipotoxic cardiomyopathy. J. Clin. Invest., 107: 813-822. DOI: 10.1172/JCI10947

Chiu, H.C., A. Kovacs, R.M. Blanton, X. Han and M. Courtois et al., 2005. Transgenic expression of fatty acid transport protein 1 in the heart causes lipotoxic cardiomyopathy. Circ. Res., 96: 225-233. DOI: 10.1161/01.RES.0000154079.20681.B9

Claycomb, W.C., N.A.J. Lanson, B.S. Stallworth, D.B. Egeland and J.B. Delcarpio et al., 1998. HL1 cells: A cardiac muscle cell line that contracts and retains phenotypic characteristics of the adult cardiomyocyte. Proc. Natl. Acad. Sci. 95: 29792984. PMID: 9501201

Der, P., J. Cui and D.K. Das, 2006. Role of lipid rafts in ceramide and nitric oxide signaling in the ischemic and preconditioned hearts. J. Mol. Cell. Cardiol., 40: 313-320. DOI: 10.1016/j.yjmcc.2005.10.005

Fauconnier, J., D.C. Andersson, S.J. Zhang, J.T. Lanner and R. Wibom et al., 2007. Effects of palmitate on $\mathrm{Ca}(2+)$ handling in adult control and ob/ob cardiomyocytes: Impact of mitochondrial reactive oxygen species. Diabetes, 56: 1136-1142. DOI: $10.2337 / \mathrm{db} 06-0739$

Feron, O. and J.L. Balligand, 2006. Caveolins and the regulation of endothelial nitric oxide synthase in the heart. Cardiovasc Res., 69: 788-797. DOI: 10.1016/j.cardiores.2005.12.014

Feron, O., F. Saldana, J.B. Michel and T. Michel, 1998. The endothelial nitric-oxide synthase-caveolin regulatory cycle. J. Biol. Chem., 273: 3125-3128. DOI: $10.1074 /$ jbc.273.6.3125

Garcia-Cardena, G., P. Oh, J. Liu, J.E. Schnitzer and W.C. Sessa, 1996. Targeting of nitric oxide synthase to endothelial cell caveolae via palmitoylation: Implications for nitric oxide signaling. Proc. Natl. Acad. Sci., 93: 6448-6453. PMID: 8692835

Gratton, J.P., P. Bernatchez and W.C. Sessa, 2004. Caveolae and caveolins in the cardiovascular system. Circ. Res., 94: 1408-1417. DOI: 10.1161/01.RES.0000129178.56294.17

He, Q., V.J. Johnson, M.F. Osuchowski and R.P. Sharma, 2004. Inhibition of serine palmitoyltransferase by myriocin, a natural mycotoxin, causes induction of c-myc in mouse liver. Mycopathologia, 157: 339-347. DOI: 10.1023/B:MYCO.0000024182.04140.95

Ingwall, J.S., 2002. ATP and the Heart. 1st Edn., Springer, Kluwer Academic Publishers, Boston, ISBN: 1402070934, pp: 244.

Johnson, V.J., Q. He, M.F. Osuchowski and R.P. Sharma. 2004. Disruption of sphingolipid homeostasis by myriocin, a mycotoxin, reduces thymic and splenic T-lymphocyte populations. Toxicology, 201: 67-75. DOI: 10.1016/j.tox.2004.04.019 
Kanai, A.J., L.L. Pearce, P.R. Clemens, L.A. Birder and M.M. Van Bibber et al., 2001. Identification of a neuronal nitric oxide synthase in isolated cardiac mitochondria using electrochemical detection. Proc. Nat. Acad. Sci., 98: 14126-14131. DOI: 10.1073/pnas.241380298

Kong, J.Y. and S.W. Rabkin, 2003. Mitochondrial effects with ceramide-induced cardiac apoptosis are different from those of palmitate. Arch. Biochem. Biophys., 412: 19-206. DOI: 10.1016/S0003-9861(03)00008-0

Kong, J.Y. and S.W. Rabkin, 2004. Reduction of palmitate-induced cardiac apoptosis by fenofibrate. Mol. Cell. Biochem. 258: 1-13. DOI: 10.1023/B:MCBI.0000012811.89386.a8

Liu, J., G. Garcia-Cardena and W.C. Sessa. 1996. Palmitoylation of endothelial nitric oxide synthase is necessary for optimal stimulated release of nitric oxide: Implications for caveolae localization. Biochemistry, 35: 13277-13281. DOI: 10.1021/bi961720e

Lowry, O.H., N.J. Rosebrough, A.L. Farr and R.J. Randall. 1951. Protein measurement with the Folin phenol reagent. J. Biol. Chem.193: 265-275. PMID: 14907713

Miller, T.A., N.K. LeBrasseur, G.M. Cote, M.P. Trucillo and D.R. Pimentel et al., 2005. Oleate prevents palmitate-induced cytotoxic stress in cardiac myocytes. Biochem. Biophys. Res. Commun., 336: 309-315. DOI: 10.1016/j.bbrc.2005.08.088

Minetti, C., F. Sotgia, C. Bruno, P. Scartezzini and P. Broda et al., 1998. Mutations in the caveolin-3 gene cause autosomal dominant limb-girdle muscular dystrophy. Nat. Genet., 18: 365-368. DOI: $10.1038 / n g 0498-365$

Mubagwa, K. and W. Flameng, 2001. Adenosine, adenosine receptors and myocardial protection: An updated overview. Cardiovasc. Res., 52: 25-39. DOI: 10.1016/S0008-6363(01)00358-3

Okamoto, T., A. Schlegel, P.E. Scherer and M.P. Lisanti, 1998. Caveolins, a family of scaffolding proteins for organizing "preassembled signaling complexes" at the plasma membrane. J. Biol. Chem., 273: 5419-5422. DOI: $10.1074 /$ jbc. 273.10 .5419
Okere, I.C., M.P. Chandler, T.A. McElfresh, J.H. Rennison and V. Sharov et al., 2006. Differential effects of saturated and unsaturated fatty acid diets on cardiomyocyte apoptosis, adipose distribution and serum leptin. Am. J. Physiol. Heart Circ. Physiol., 291: 38-44. DOI: 10.1152/ajpheart. 01295.2005

Pinz, I., S.D. Wax, P. Anderson and J.S. Ingwall. 2008. Low over-expression of TNFalpha in the mouse heart increases contractile performance via TNFR1. J. Cell Biochem., 105: 99-107. PMID: 18452158

Song, K.S., S. Li, T. Okamoto, L.A. Quilliam and M. Sargiacomo et al., 1996. Co-purification and direct interaction of Ras with caveolin, an integral membrane protein of caveolae microdomains. Detergent-free purification of caveolae microdomains. J. Biol. Chem., 271: 9690-9697. DOI: 10.1074/jbc.271.16.9690

Tsang, M.Y., S.E. Cowie and S.W. Rabkin, 2004. Palmitate increases nitric oxide synthase activity that is involved in palmitate-induced cell death in cardiomyocytes. Nitric Oxide, 10: 11-19. DOI: 10.1016/j.niox.2004.01.005

Williams, T.M. and M.P. Lisanti, 2004. The caveolin proteins. Genome. Biol., 5: 214-214.

Woodman, S.E., D.S. Park, A.W. Cohen, M.W. Cheung and M. Chandra et al., 2002. Caveolin-3 knockout mice develop a progressive cardiomyopathy and show hyperactivation of the p42/44 MAPK cascade. J. Biol. Chem., 277: 38988-38997. DOI: 10.1074/jbc.M205511200

Yagyu, H., G. Chen, M. Yokoyama, K. Hirata and A. Augustus et al., 2003. Lipoprotein lipase (LpL) on the surface of cardiomyocytes increases lipid uptake and produces a cardiomyopathy. J. Clin. Invest., 111: 419-426. DOI: 10.1172/JCI16751

Young, M.E., P.H. Guthrie, P. Razeghi, B. Leighton and S. Abbasi et al., 2002. Impaired long-chain fatty acid oxidation and contractile dysfunction in the obese zucker rat heart. Diabetes, 51: 25872595. DOI: $10.2337 /$ diabetes.51.8.2587

Zhou, Y.T., P. Grayburn, A. Karim, M. Shimabukuro and M. Higa et al., 2000. Lipotoxic heart disease in obese rats: Implications for human obesity. Proc. Natl. Acad. Sci., 97: 1784-1789. DOI: 10.1073/pnas.97.4.1784 